Effects of neutral distribution and external magnetic field on plasma momentum in electrodeless plasma thrusters

Kazuki Takase, Kazunori Takahashi, and Yoshinori Takao

Citation: Physics of Plasmas 25, 023507 (2018);

View online: https://doi.org/10.1063/1.5015937

View Table of Contents: http://aip.scitation.org/toc/php/25/2

Published by the American Institute of Physics

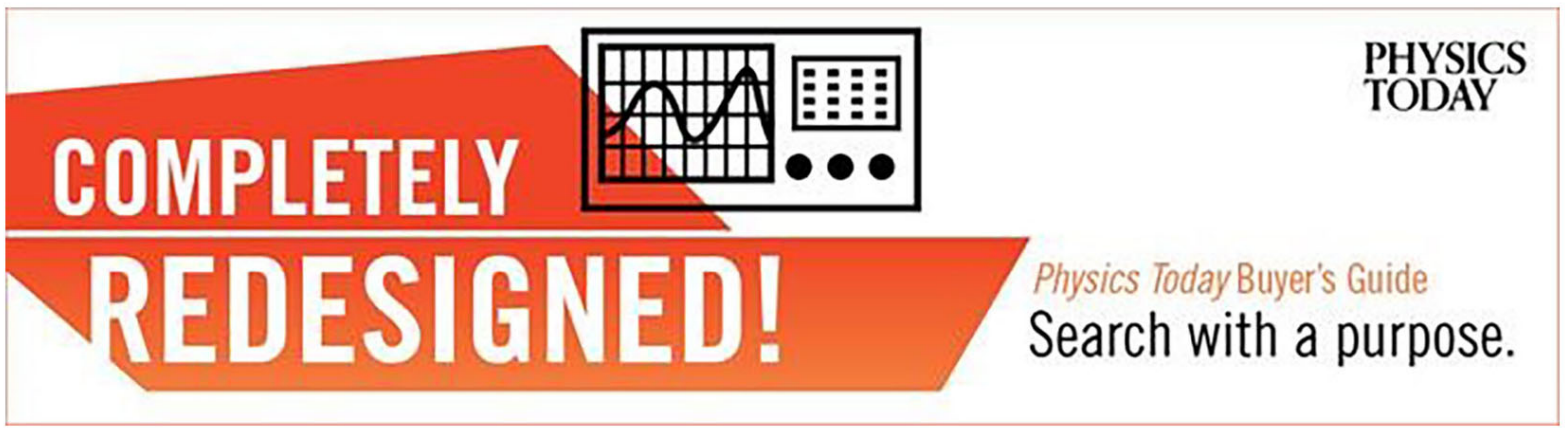




\title{
Effects of neutral distribution and external magnetic field on plasma momentum in electrodeless plasma thrusters
}

\author{
Kazuki Takase, ${ }^{1}$ Kazunori Takahashi, ${ }^{2}$ and Yoshinori Takao ${ }^{3, a)}$ \\ ${ }^{1}$ Department of Systems Integration, Yokohama National University, Yokohama 240-8501, Japan \\ ${ }^{2}$ Department of Electrical Engineering, Tohoku University, Sendai 980-8579, Japan \\ ${ }^{3}$ Division of Systems Research, Yokohama National University, Yokohama 240-8501, Japan
}

(Received 15 November 2017; accepted 22 January 2018; published online 6 February 2018)

\begin{abstract}
The effects of neutral distribution and an external magnetic field on plasma distribution and thruster performance are numerically investigated using a particle-in-cell simulation with Monte Carlo collisions (PIC-MCC) and the direct simulation Monte Carlo (DSMC) method. The modeled thruster consists of a quartz tube $1 \mathrm{~cm}$ in diameter and $3 \mathrm{~cm}$ in length, where a double-turn rf loop antenna is wound at the center of the tube and a solenoid is placed between the loop antenna and the downstream tube exit. A xenon propellant is introduced from both the upstream and downstream sides of the thruster, and the flow rates are varied while maintaining the total gas flow rate of $30 \mu \mathrm{g} / \mathrm{s}$. The PIC-MCC calculations have been conducted using the neutral distribution obtained from the DSMC calculations, which were applied with different strengths of the magnetic field. The numerical results show that both the downstream gas injection and the external magnetic field with a maximum strength near the thruster exit lead to a shift of the plasma density peak from the upstream to the downstream side. Consequently, a larger total thrust is obtained when increasing the downstream gas injection and the magnetic field strength, which qualitatively agrees with a previous experiment using a helicon plasma source. Published by AIP Publishing.
\end{abstract}

https://doi.org/10.1063/1.5015937

\section{INTRODUCTION}

Since efficient helicon plasma production was discovered in the 1970 s, many studies related to its formation mechanism and applications have been performed. ${ }^{1-3}$ Helicon plasma sources have some advantages such as highdensity plasma production with relatively low rf power and are utilized in various fields such as plasma processing ${ }^{4-6}$ and space propulsion. ${ }^{7-12}$ Recently in the field of space engineering, high-power electric thrusters have become one of the featured topics since the development of all-electric satellites around the world has been advanced to reduce their weight to achieve low launch costs. Although electric propulsion devices require long-term operation owing to their low thrust compared with conventional chemical ones, electric propulsion is expected to be one of the candidates for large cargo transportation and deep space exploration.

In order to achieve such long-term missions, it is considered that the development of an electric propulsion system with no electrode is necessary because the electrode erosion typically determines the lifetime of the propulsion devices. In particular, helicon plasma thrusters (HPTs) consisting of a helicon plasma source and a magnetic nozzle are attracting attention since no electrodes are required for plasma generation and acceleration. However, the propulsion performance of HPTs is still relatively low compared with the conventional thrusters. A recent experiment ${ }^{7}$ and numerical simulation ${ }^{13}$ have implied that neutral depletion inside the thrusters causes axially asymmetric plasma distribution and axial momentum loss, i.e., the

\footnotetext{
${ }^{\text {a)} E-m a i l: ~ t a k a o-y o s h i n o r i-y k @ y n u . j p ~}$
}

non-negligible axial force exerted on the lateral wall in the opposite direction to the thrust. The neutral depletion is further enhanced when the rf power for plasma production is increased as in Ref. 14.

The structural modification of the plasma due to the neutral depletion phenomenon is a common problem not only in thrusters but also in terrestrial plasma devices. ${ }^{15,16}$ Actually, neutral depletion has been observed in a number of experiments and analyses, ${ }^{14}$ and numerical studies have proposed plasma structural formation and transport in such situations. ${ }^{17,18}$ Takahashi et al. ${ }^{19}$ have demonstrated that neutral gas injection from not only the upstream side but also the downstream side of the thruster inhibits the formation of the upstream density peak, resulting in increases in the downstream plasma density and the thrust. Hence, the neutral density profile seems to significantly affect the thruster performance due to the structural formation and momentum transport inside the source. After more addressing the axial density profile and the axial momentum loss, these are observed to be changed by the magnetic field configuration. ${ }^{20}$

In a previous study, ${ }^{13}$ the axial momentum loss was numerically demonstrated in a small inductively coupled plasma source with no magnetic field using particle-in-cell simulations with Monte Carlo collisions (PIC-MCC), where the neutral density profile was artificially changed. Here, PIC-MCC including the magnetic field is combined with a direct simulation Monte Carlo (DSMC) method, which can yield a neutral density profile, since the experiments have shown the importance of both the magnetic field configuration and the location of the gas injection port. The plasma structure and the imparted force components on the axial 
back wall and the radial source wall are numerically calculated, with the neutral profile being changed by injecting the gas from either the upstream or the downstream side with and without a magnetic field. Since the combination of PIC and DSMC simulations is a very time-consuming calculation, the neutral depletion effect is not included to simplify the model in this study. Instead, this work investigates and discusses the effects of various combinations of neutral profiles and magnetic field on the thrust components, where the application of magnetic fields and injection of the gas from the downstream side result in a shift of the plasma density peak to the thruster downstream side, while momentum loss to the lateral wall is inhibited.

\section{NUMERICAL MODEL}

Figures 1(a) and 1(b) show the schematics of the calculation model for the DSMC and PIC-MCC simulations, respectively, where a 0.7 -mm-thick cylindrical dielectric quartz tube and a double-turn rf loop antenna located at the center of the tube are employed. The rf frequency is set at $80 \mathrm{MHz}$, and the inner radius and the length are 0.5 and $3 \mathrm{~cm}$, respectively; these values are roughly one-sixth of those used in the recent experimental configuration. ${ }^{7}$ This size reduction is due to the limitation of the computational performance to obtain the solution within an acceptable calculation time. Moreover, for the same reason, metal plates for both axial ends of the quartz tube are employed for the PIC-MCC simulation as shown in Fig. 1(b), where the calculation speed can be accelerated using a fast Fourier transformation to solve Poisson's equation. ${ }^{21}$ These conditions and configuration are the same as those employed in the previous study. ${ }^{13}$ An open boundary is set at $z=3.0 \mathrm{~cm}$ for the DSMC simulation to calculate the neutral density profile, while the solenoid coil is located at $z=2.4 \mathrm{~cm}$ in the closed system for
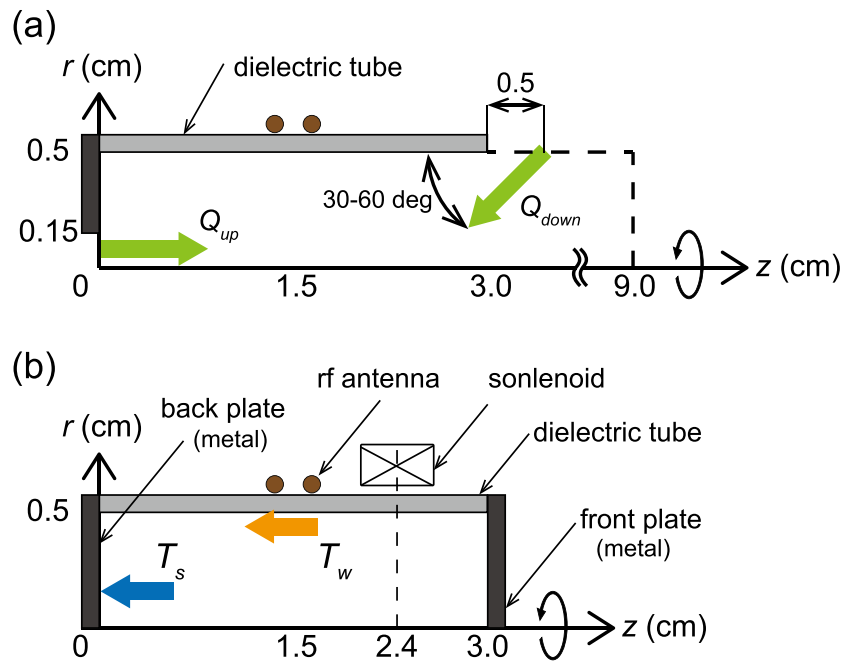

FIG. 1. Schematic diagrams of the calculation areas for (a) the DSMC and (b) PIC-MCC simulations. Neutral gas injection points from the upstream $Q_{u p}$ and downstream $Q_{\text {down }}$ are also illustrated in (a), and the positive direction of each thrust force $\left(T_{s}\right.$ and $\left.T_{w}\right)$ is also defined in (b). While an open boundary at the thruster exit is employed in the DSMC simulations, a fully closed boundary is used in the PIC-MCC calculations to reduce the calculation time. the PIC-MCC simulation. The grid spacing for both the DSMC and the PIC-MCC is set at regular intervals of 0.1 $\mathrm{mm}$, and cylindrical coordinates $(r-z)$ are employed; xenon is utilized as the working gas.

As shown in Fig. 1(b), we defined the forces on the back plate $T_{s}$ and on the lateral wall $T_{w}$ as positive in the negative $z$-direction in order to evaluate the propulsion performance; these forces are defined according to the theoretical models $^{11,22,23}$ as

$$
\begin{gathered}
T_{s}=2 \pi \int_{0}^{r_{s}} r p_{e}\left(r, z_{0}\right) d r \\
T_{w}=-2 \pi \int_{0}^{z_{e}} r_{s} m n\left(r_{s}, z\right) u_{r}\left(r_{s}, z\right) u_{z}\left(r_{s}, z\right) d z,
\end{gathered}
$$

where $r_{\mathrm{s}}, z_{e}, p_{e}, z_{0}, m, n, u_{r}$, and $u_{z}$ are the radius of the lateral wall, position of the thruster exit ( $3 \mathrm{~cm}$ in this case), electron pressure, maximum pressure position, ion mass, ion density, and radial and axial ion velocities, respectively. Since $T_{s}$ is obtained by integrating the maximum electron pressure over the cross-section at $z=z_{0}, T_{s}$ represents the pressure force on the back plate, implying that the electron pressure is converted into ion momentum toward the back plate through sheath acceleration due to the potential drop. ${ }^{24}$ Equation (2) indicates that the axial momentum $m u_{z}$ is delivered to the lateral wall by ions lost radially with the flux $n u_{r}$, which was previously neglected ${ }^{17,25}$ until one of the authors experimentally showed a significant loss of the axial plasma momentum to the lateral wall. ${ }^{7}$ These forces are obtained directly by calculating the momentum of the ions colliding with the wall boundaries of the simulation area, where the force imparted to the wall is underestimated owing to the neglect of neutral effects as in the previous study. ${ }^{13}$ Although the Lorentz force onto the magnetic nozzle is also a major thrust component when a strong magnetic field is applied, ${ }^{9}$ this effect cannot be taken into account for the present closed plasma source. In this study, we focus on the ion motion towards the back and lateral walls, and its dependence on the neutral distribution and the magnetic field is investigated.

To represent various neutral distributions, neutral gas with a temperature of $300 \mathrm{~K}$ is introduced from both the upstream and downstream regions as shown in Fig. 1(a). The gas flow rates from the upstream and downstream regions are defined as $Q_{u p}$ and $Q_{\text {down }}$, respectively, and several injection ratios are employed, while the total gas flow rate $Q_{\text {total }}$ is maintained at $30 \mu \mathrm{g} / \mathrm{s}$. This value is set so that the pressure value at the left end of the source tube is similar to the one used in the previous study. ${ }^{13}$ The upstream gas injection is performed from the center of the back plate as conducted in the recent experiment ${ }^{19}$ while the downstream gas injection is carried out from the ring-shaped slit because of the axisymmetric cylindrical coordinates in the present simulations. The location of the downstream gas injection is set at the radial boundary $(r=0.57 \mathrm{~cm})$ and $0.5 \mathrm{~cm}$ downstream of the thruster exit, where the gas injection angle is $30^{\circ}-60^{\circ}$ so that all the neutral particles injected can reach inside the quartz tube. We choose the ratios of the downstream gas flow rates to the total gas flow rates, $Q_{\text {down }} / Q_{\text {total }}$, as $0 \%, 5 \%, 25 \%$, 
$50 \%, 75 \%$, and $95 \%$. Neutral atoms are assumed to be diffusely reflected on the thruster wall with Maxwellian velocities at a temperature of $300 \mathrm{~K}$. Here, collisions between neutral atoms are not taken into account for the following reason. The gas flow rate of $30 \mu \mathrm{g} / \mathrm{s}$ is equivalent to the average pressure of $0.96 \mathrm{mT}$ Torr inside the thruster under a steady state for only the upstream gas injection, where the mean free path is estimated to be $6.1 \mathrm{~cm}$ at $300 \mathrm{~K}$. This value is larger than the thruster length, and thus, collisions would be negligible.

Four types of magnetic field configurations, namely 0 , 50,100 , and $200 \mathrm{G}$ at the solenoid center on the $z$-axis, are tested in the present study. Figure 2 shows the contour distribution of the external magnetic field strength $B_{e x}$ with the magnetic field lines (solid lines) and the distribution of the axial magnetic field $B_{z}$ on the $z$-axis for $B_{e x}$ values of $200 \mathrm{G}$. $B_{e x}$ and $B_{z}$ decrease gradually with increases in the distance from the solenoid center. It should be noted that the maximum external magnetic field is limited to about $200 \mathrm{G}$ or less since the simulation becomes unstable under the high magnetic field due to the high electron density and the resultant short Debye length, which violates the stable condition for the PIC-MCC simulations.

In the previous simulation, the neutral density distribution was uniform or neutral depletion was artificially introduced, and no external magnetic field was imposed. The present PIC-MCC model is the same as the previous one, ${ }^{13}$ except that the background neutral distribution is given by the result obtained from the DSMC calculation and the external magnetic field induced by the solenoid coil is added.

The simulations have been conducted under the following assumptions: (i) Only singly ionized xenon and electrons

(a)

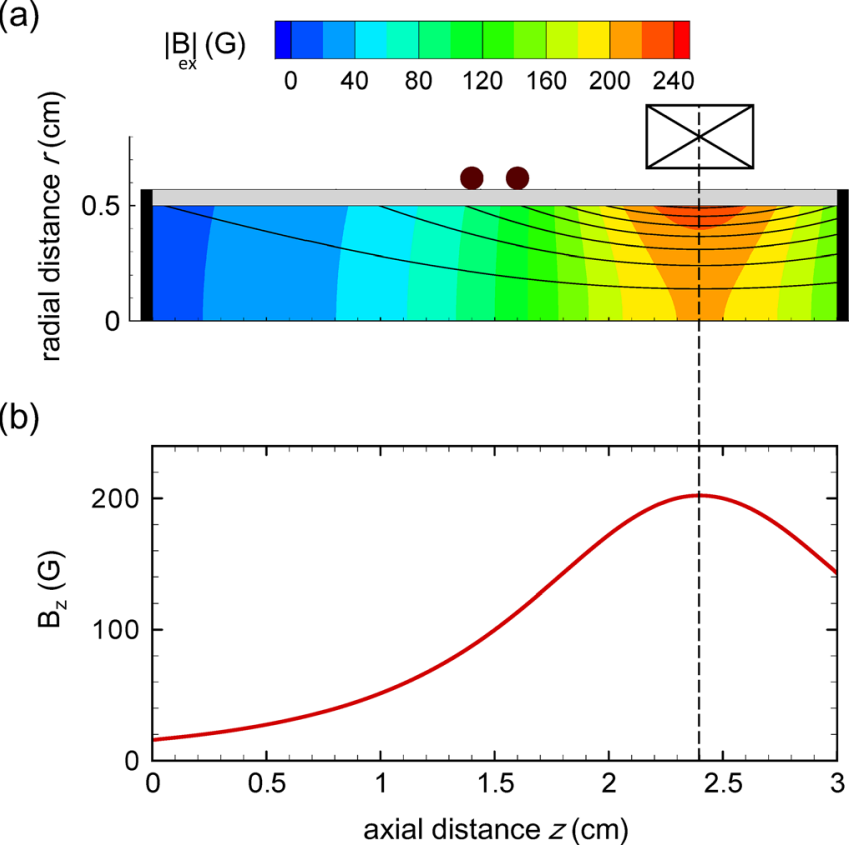

FIG. 2. (a) The distribution of the calculated external magnetic field strength (contours) with magnetic field lines (solid lines). (b) Axial profile of the magnetic field strength on the $z$-axis for $B_{\mathrm{ex}}=200 \mathrm{G}$. The dashed line plotted in the figures indicates the position of the solenoid center $(z=2.4 \mathrm{~cm})$ as a visual guide. are treated as particles. (ii) The collisional reactions considered here are elastic, excitation, and ionization collisions for electrons and elastic and charge exchange collisions for ions, where the cross-section dataset is the same as that used in an earlier study. $^{26}$ (iii) The motion of excited-state atoms and Coulomb collisions (electron-electron, ion-ion, and electronion) are not taken into account. (iv) The neutral distributions obtained from the DSMC simulation are fixed on the grids, and no dynamics of the neutrals are taken into account in the PIC-MCC simulations, indicating that these two simulations have been conducted independently.

The PIC-MCC model consists of the electromagnetic equation for the rf-induced azimuthal electric field, the Poisson equation for the electrostatic field due to the space charge, the equation of motion, and the collisions between charged particles and neutrals. To couple the rf power to the plasma, assuming that all wave quantities vary harmonically in time as $e^{i \omega t}$, where $i$ is the square root of -1 and $t$ is the time, and the rf loop antenna is composed of two concentric rings, and thus, the rf-induced electric field has only the azimuthal component, we solve the following electromagnetic equation: ${ }^{21,27-30}$

$$
\left(\frac{\partial^{2}}{\partial r^{2}}+\frac{1}{r} \frac{\partial}{\partial r}+\varepsilon_{0} \mu_{0} \omega^{2}-\frac{1}{r^{2}}+\frac{\partial^{2}}{\partial z^{2}}\right) \tilde{E}_{\theta}=i \omega \mu_{0} \tilde{j}_{\theta},
$$

where $\varepsilon_{0}, \mu_{0}, \omega, \tilde{E}_{\theta}$, and $\tilde{j}_{\theta}$ are the electric permittivity of a vacuum, the magnetic permeability of a vacuum, rf angular frequency, complex amplitudes of the $\mathrm{rf}$ induced electric field, and the plasma current density, respectively. It should be noted that we treat the plasma as inductively coupled discharges and the plasma current density $\tilde{j}_{\theta}$ is directly taken from the following electron trajectories obtained by the equation of motion, not from the cold plasma approximation. Thus, this calculation is fully kinetic, and no assumptions are required about the mechanism of electron heating: the effect of non-collisional heating is also automatically taken into account. After the azimuthal electric field is determined by Eq. (3), the time-varying magnetic fields $\left(B_{r}, B_{z}\right)$ are obtained by Faraday's law. These magnetic fields are superimposed on the external magnetic field of the solenoid coil, i.e., the values of $B_{r}+B_{e x, r}$ and $B_{z}+B_{e x, z}$ are used for the equation of motion. The $\mathrm{rf}$ electromagnetic fields are updated every $10 \mathrm{rf}$ cycles to reduce the calculation cost. These calculation methods imply that helicon wave propagation is not taken into account in the current model although the external magnetic field is applied. The equation of motion for the charged particles is solved explicitly by a time-centered leapfrog method for time integration and the Buneman-Boris method for the $\boldsymbol{v} \times \boldsymbol{B}$ rotation term. ${ }^{31}$ For the particle collision scheme in MCC, we employ the null-collision method to reduce the calculation time. ${ }^{32}$ Compared to the previous study, ${ }^{13}$ the distribution of the neutral particle obtained from the DSMC calculation is also taken into account to obtain the collision probability in MCC.

Initially, spatially uniform ions and electrons with Maxwellian velocities are placed in the simulation area, where the total number of electron and ion superparticles is set at about 1500000 , respectively. Here, we use a spatially uniform 


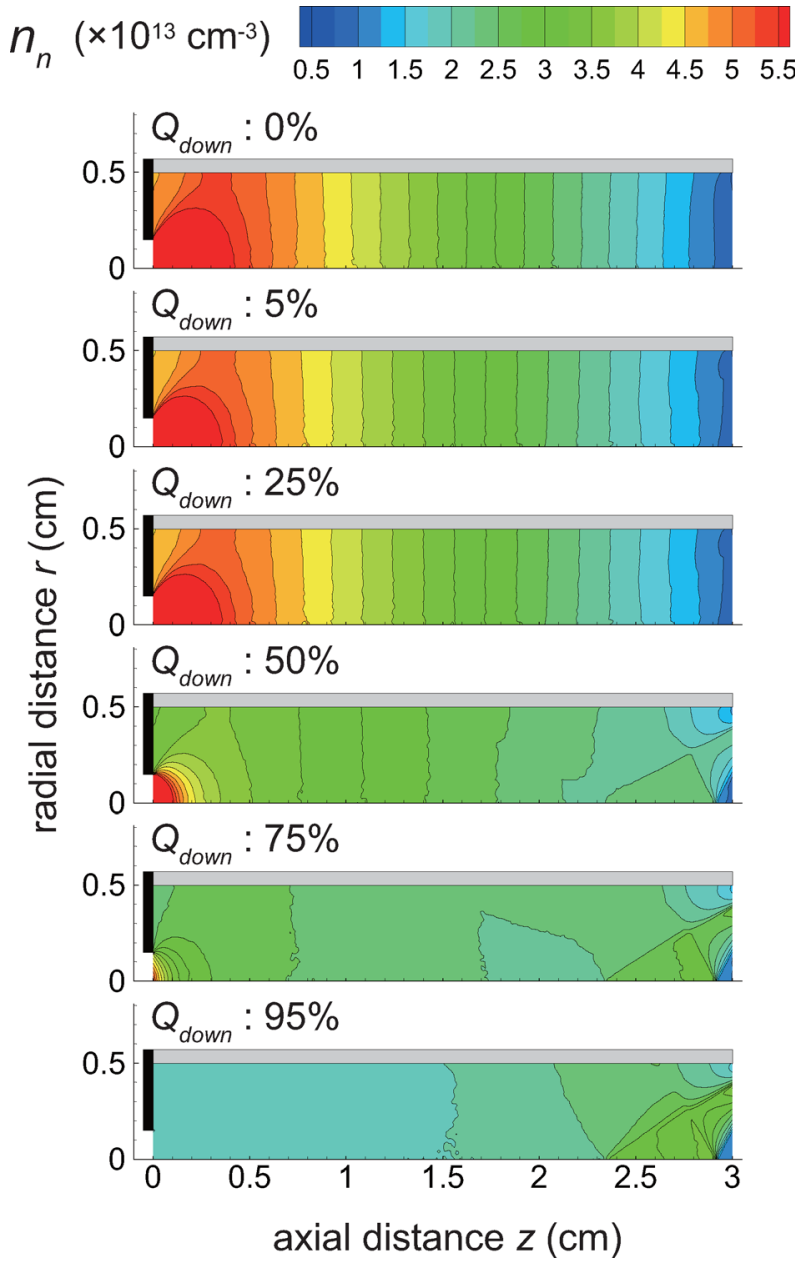

FIG. 3. Two-dimensional distributions of the neutrals in a steady state for each downstream gas injection ratio $\left(Q_{\text {down }} / Q_{\text {total }}\right)$. Here, only the results inside the thruster are plotted.

weight of superparticles for the cylindrical coordinates with a general weighting scheme presented by Verboncoeur ${ }^{33}$ to eliminate a systematic error in the number density on the $z$-axis $(r=0)$. The numerical time step for electrons is taken to be $5.0 \times 10^{-12} \mathrm{~s}$, which is sufficient to resolve the electron plasma frequency and gyration frequency. The power absorption in the plasma is used as an input parameter, where the power absorption is obtained by calculating the change in kinetic energy of electrons and ions before and after each charged particle is moved on integrating the equation of motion. ${ }^{34}$ We rescale the two-turn antenna current to yield the specified power absorption until the steady-state solution is obtained, which requires roughly two million time steps (10 $\mu \mathrm{s})$. The macroscopic parameters, such as the electron density and temperature and plasma potential, are determined by averaging over $10 \mu$ s after a steady state is reached. To obtain the above parameters in the PIC simulations, a typical example of the total computational time is about $1-2$ weeks on a workstation with four 3.7-GHz processors, where the code is parallelized only for the motion of charged particles and MCC. For the DSMC simulations, the total number of superparticles is about five million, and it takes a few hours to reach a steady state with a single core processor.

\section{RESULTS AND DISCUSSION}

Figure 3 shows the two-dimensional distributions of the neutral density obtained by the DSMC simulation for various downstream gas injection ratios. With the increasing $Q_{\text {down }}$ ratio, the maximum neutral density near the back wall decreases and its density gradient becomes gentle. When 95\% of neutrals are introduced from the downstream side, the location of the neutral density peak shifts to the downstream region. The neutral density averaged inside the thruster tube also decreases: $3.52 \times 10^{13}, 3.42 \times 10^{13}$, $3.13 \times 10^{13}, 2.80 \times 10^{13}, 2.41 \times 10^{13}$, and $2.12 \times 10^{13} \mathrm{~cm}^{-3}$ in the ascending order of the $Q_{\text {down }}$ ratio because some of the neutrals injected from the downstream are diffusely reflected on the lateral wall and exit from the thruster without reaching the back plate $(z=0 \mathrm{~cm})$. This tendency implies that downstream gas injection might result in low propellant utilization efficiency.

\section{A. Effects of the external magnetic field}

Under the neutral distributions shown in Fig. 3, we first analyzed the motion of charged particles with and without applied magnetic fields in the PIC-MCC simulations at the absorbed power of $1 \mathrm{~W}$. Figure 4 shows the two-dimensional distributions of the electron density, the plasma potential, and the electron temperature in a steady state for each $B_{e x}$, where the neutrals are introduced from the upstream side only $\left(Q_{\text {down }} / Q_{\text {total }}=0 \%\right)$. When setting the applied magnetic field to zero, upstream-peaking profiles of the plasma density and potential are clearly observed and seem to originate from the increased ionization frequency due to the upstreampeaking profile of the neutral density as shown in Fig. 3 at $Q_{\text {down }} / Q_{\text {total }}=0 \%$. This potential profile leads to the presence of the electric field in the upstream region, where the ions accelerated into the positive $z$-direction travel a long distance inside the plasma source and are lost to the lateral wall; thus, their axial momentum is delivered to the lateral wall as proposed in Ref. 7. Here, the calculated $T_{w}$ is about $-6.4 \mu \mathrm{N}$, which is non-negligible compared with the $T_{s}$ of $18.7 \mu \mathrm{N}$ in this case. This tendency is in qualitative agreement with the previous experiment, where a large negative $T_{w}$ was obtained when the solenoid coil current was set to zero. ${ }^{7,9}$ On increasing $B_{e x}$, the plasma distribution changes from an upstream-peaking profile to a downstream-peaking one, where the maximum plasma density increases and the peak plasma potential decreases.

For the larger magnetic field strength, the electrons are tied to the magnetic field lines converging at the magnetic nozzle throat, resulting in both inhibition of the electron loss to the lateral wall and enhancement of the local ionization process due to the increase in the electron density, which are considered to contribute to the density peak at around $z=2 \mathrm{~cm}$. In addition, the positive values of $T_{w}=7.8 \mu \mathrm{N}$ and $22.7 \mu \mathrm{N}$ for $B_{e x}=100$ and $200 \mathrm{G}$, respectively, are obtained (as shown in and discussed with Fig. 7 later), while the previous experiment has shown a value of $T_{w}$ close to zero 9 owing to the axially symmetric plasma profile for the high magnetic field condition. The discrepancy between the simulation and the experiment is probably due to different conditions. In the 
(a) $n_{\mathrm{e}}\left(\times 10^{11} \mathrm{~cm}^{-3}\right)$
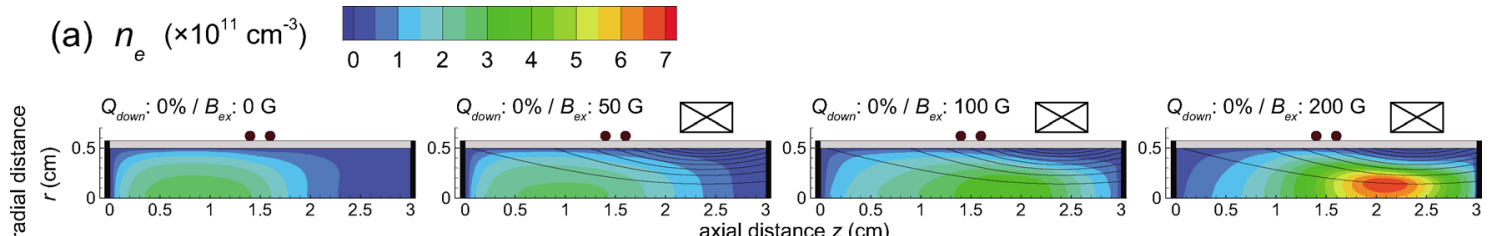

(b) $\phi(V)$
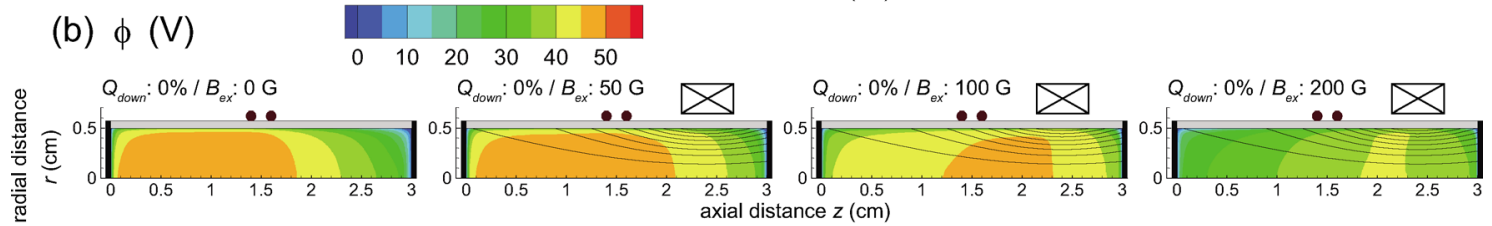

(c) $T_{e}(\mathrm{eV})$
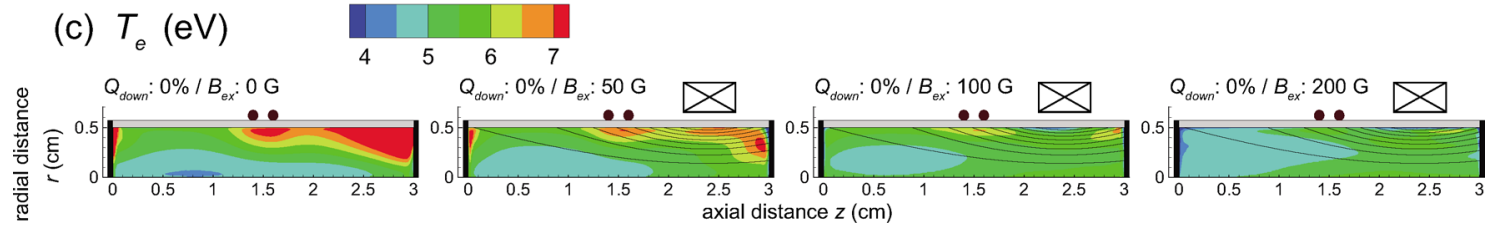

FIG. 4. Two-dimensional distributions of (a) the electron density, (b) plasma potential, and (c) electron temperature in a steady state (contours) at each value of $B_{\text {ex }}$, together with the magnetic field lines (solid lines) for $B_{e x}=50,100$, and $200 \mathrm{G}$, where the neutrals are introduced from the upstream side only $\left(Q_{d o w n} / Q_{\text {total }}=0 \%\right.$ ).

present simulation, since the size of the plasma source tube is reduced by one-sixth, the gas pressure inside the source tube is increased to maintain the equal ratio of the mean free path to the tube size. Moreover, the rf plasma discharge is sustained by inductive coupling, not by helicon waves. These differences and not taking the neutral depletion effect into account result in the localized peak of the plasma density at the downstream side when applying a stronger magnetic field. Nonetheless, it is clearly indicated that applying a magnetic field contributes to the changes in the plasma profile and the thrust performance without downstream gas injection.

\section{B. Competition between the magnetic field and the neutral profile effects}

Figure 5 shows the two-dimensional distributions of the electron density, the plasma potential, and the electron temperature for various $B_{e x}$ and $Q_{\text {down }}$ ratios. As shown in Fig. $5(\mathrm{a})$, the numerical results indicate two clear tendencies. First, the increase in $Q_{\text {down }}$ leads to shifts of the peak density to the downstream side for all values of $B_{e x}$. Second, the increase in $B_{e x}$ results in increasing peak values of the plasma density and additional shifts to beneath the solenoid coil for all $Q_{\text {down }}$ ratios. These results indicate that not only the increase in $B_{e x}$ as described above but also the increase in the $Q_{\text {down }}$ ratio is an effective method of achieving the maximum plasma density in the downstream regions. On the other hand, only a small axial shift of the density peak on changing the $Q_{\text {down }}$ ratio is observed for the high magnetic field of $200 \mathrm{G}$, compared with the cases of weak magnetic fields. This shows that the most downstream position of the maximum density is close to the magnetic throat even when injecting the gas from the downstream open source exit. Therefore, the downstream gas injection has a small influence on the plasma distributions under stronger magnetic field conditions.

The potential profiles are similar to the electron density profiles, where the shifts of the peak potential toward the downstream regions are observed with increasing values of $B_{\text {ex }}$ and the $Q_{\text {down }}$ ratio. When the maximum potential exists near the thruster exit, the ions accelerated in the negative $z$ direction can travel a longer distance inside the source, resulting in the positive $T_{w}$. This tendency is clearly seen in Fig. 6, where the axial profiles of the calculated force densities on the lateral wall in the axial direction for each value of $Q_{\text {down }} / Q_{\text {total }}$ and $B_{e x}$ are plotted. Here, the positive direction of $T_{w}$ is defined as in Fig. 1(b), and $A$ represents the dimensions of the surface area of the lateral wall. The net force of $T_{w}$ integrating $T_{w} / A$ over the surface area of the source tube results in more positive values when $B_{e x}$ and the $Q_{\text {down }}$ ratio are increased.

The plasma potential tends to increase with increases in the $Q_{\text {down }}$ ratio and to decrease with increases in $B_{e x}$. Such potential distributions are related to the distribution of the electron temperature as shown in Fig. 5(c), where higher electron temperatures are obtained for higher $Q_{\text {down }}$ ratios and lower electron temperatures are observed for higher $B_{e x}$ values. As shown in Fig. 2, the neutral density decreases with increases in the $Q_{\text {down }}$ ratio. In regions of lower neutral density, fewer ionization collisions occur, and thus, higher electron temperatures are obtained. When $B_{e x}$ increases, electrons are confined by the magnetic field and the electron loss to the wall is inhibited, leading to the increase in the electron density without a large number of high-energy electrons. Since the absorbed power in the plasma is fixed at $1 \mathrm{~W}$ under all the calculation conditions in the present simulations, the increase in electron density does not require a high rf antenna current to absorb the power of $1 \mathrm{~W},{ }^{35}$ leading to a lower electron temperature.

The calculated thrust components, $T_{w}$ and $T_{s}$, are plotted in Figs. 7(a) and 7(b) as functions of the downstream gas injection ratio $Q_{\text {down }} / Q_{\text {total }}$ for each $B_{\text {ex }}$. With increases in both the $Q_{d o w n}$ ratio and $B_{e x}, T_{w}$ has larger values. For $B_{e x}$ values of 0 and $50 \mathrm{G}$ at lower $Q_{\text {down }}$ ratios, $T_{w}$ shows negative values that indicate momentum loss to the lateral wall, while positive values are obtained at higher $Q_{\text {down }}$ ratios. The negative values of $T_{w}$ are obtained for the upstreampeaking plasma profile, while the positive values of $T_{w}$ result from the axially asymmetric plasma distribution that has a 
(a) $n_{e}\left(\times 10^{11} \mathrm{~cm}^{-3}\right) \quad \begin{array}{lllllllll}0 & 1 & 2 & 3 & 4 & 5 & 6 & 7\end{array}$

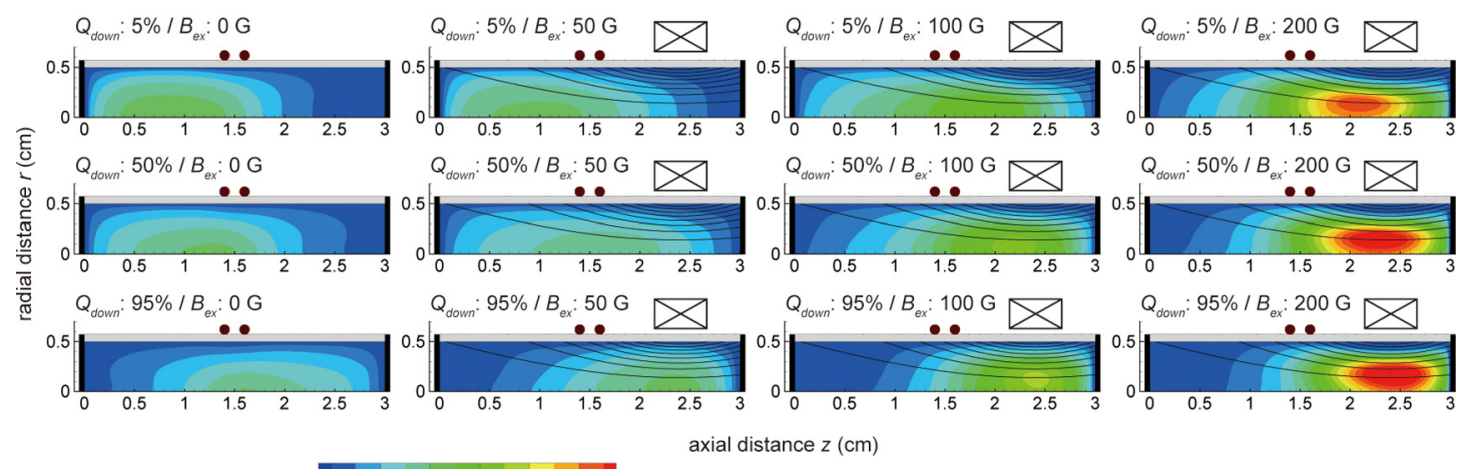

(b) $\phi(V)$
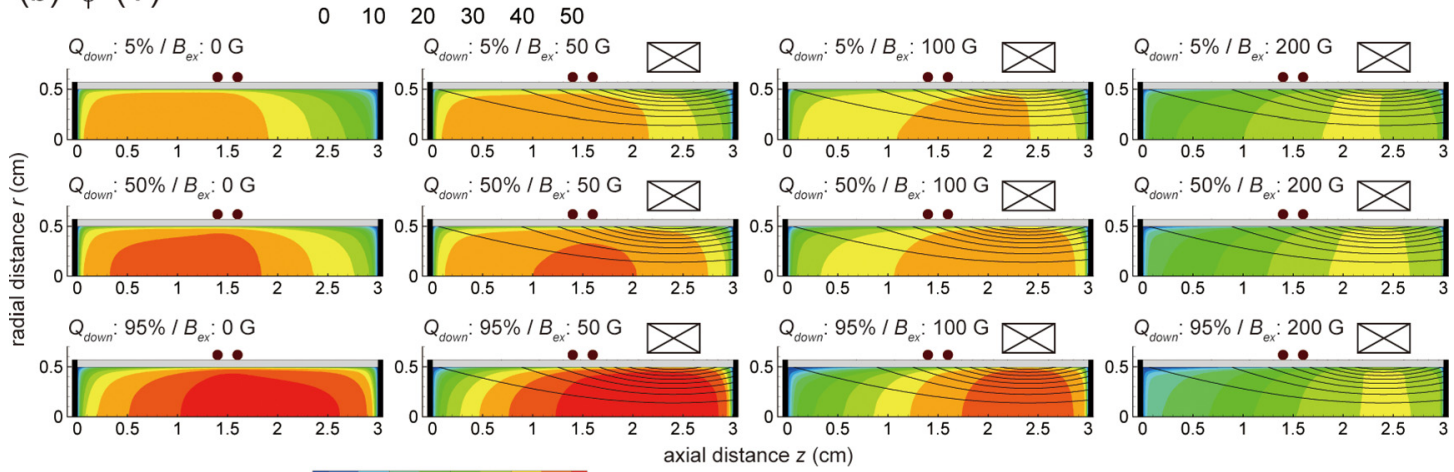

(c) $T_{e}(\mathrm{eV})$
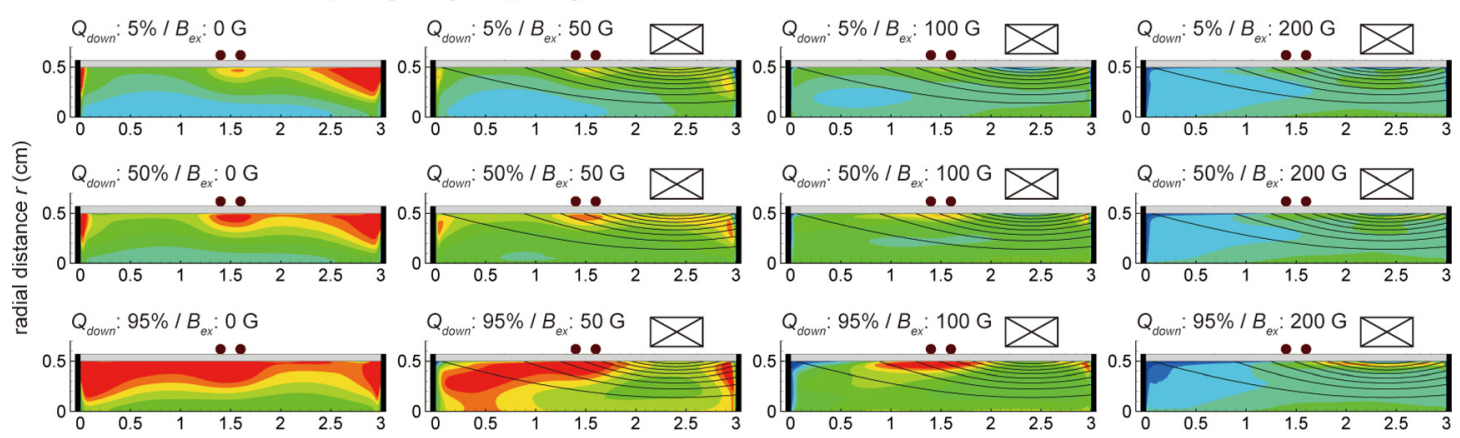

FIG. 5. Two-dimensional distributions of (a) the electron density, (b) plasma potential, and (c) electron temperature in a steady state (contours) at each value of $B_{e x}$, together with the magnetic field lines (solid lines) for $B_{e x}=50,100$, and $200 \mathrm{G}$, where only the results at $Q_{\text {down }} / Q_{\text {total }}=5 \%, 50 \%$, and $95 \%$ are selected and shown.

peak in the downstream side as compared with Fig. 5. Therefore, these results clearly show that downstream gas injection improves the axial momentum loss to the lateral wall. For $B_{e x}$ values of 100 and $200 \mathrm{G}, T_{w}$ also increases with increases in the $Q_{\text {down }}$ ratio although its rate of increase becomes smaller than that under lower $B_{e x}$ conditions. This small increase in $T_{w}$ for higher $B_{e x}$ is due to the small shift of the peak plasma profile as shown in Fig. 5. Moreover, $T_{w}$ also increases with increases in $B_{e x}$ for any $Q_{\text {down }}$ ratio. Whereas the increase in $B_{e x}$ leads to the decrease in the peak potential, high-density plasma near the solenoid coil at larger values of $B_{e x}$ makes the dominant contribution to the increase in $T_{w}$ because the thrust is derived from the momentum flux (the momentum $m u_{z}$ multiplied by the ion flux $n u_{r}$ ).

In contrast to $T_{w}, T_{s}$ decreases monotonically with increases in the $Q_{\text {down }}$ ratio regardless of the values of $B_{e x}$. These results are due to the shifts in the plasma density distributions as shown in Fig. 5, where the shifts of the peak density to the downstream side cause the decrease in density near the back plate. On increasing $B_{e x}, T_{s}$ becomes insensitive to the change in the $Q_{\text {down }}$ ratio, similarly to the tendency of $T_{w}$, which also results from the small shifts for higher $B_{e x}$ as described above.

Figure 7(c) shows the total thrust as a function of the $Q_{\text {down }}$ ratio for each value of $B_{e x}$, where the total thrust is defined as the sum of $T_{w}$ and $T_{s}$ in our simulations (not including the Lorentz force on the external magnetic field modeled in Ref. 9). The results show that the total thrust has different tendencies depending on $B_{e x}$. For $B_{e x}$ values of 0 and $50 \mathrm{G}$, the total thrust decreases with the increase in the $Q_{\text {down }}$ ratio, while it is almost constant for a $B_{e x}$ of $100 \mathrm{G}$. Since the neutral density decreases with increases in the $Q_{\text {down }}$ ratio as shown in Fig. 2, downstream gas injection has a negative influence on the total thrust when $B_{e x}$ is low. On 

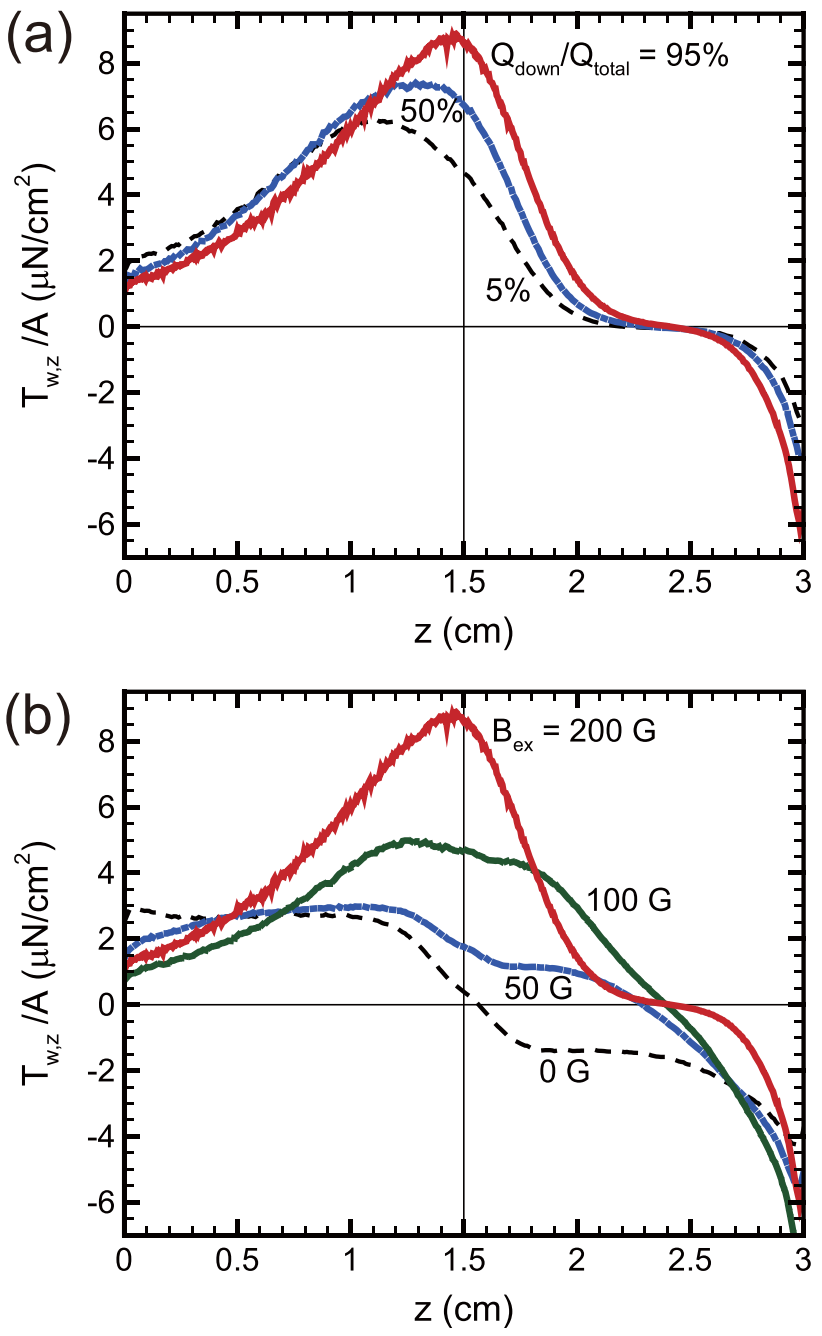

FIG. 6. Axial profiles of the calculated force densities on the lateral wall in the axial direction $\left(T_{w} / A\right.$ ) (a) for $Q_{\text {down }} / Q_{\text {total }}=5 \%, 50 \%$, and $95 \%$ at $B_{\mathrm{ex}}=200 \mathrm{G}$ and (b) for $B_{\mathrm{ex}}=0,50,100$, and $200 \mathrm{G}$ at $Q_{\text {down }} / Q_{\text {total }}=95 \%$. Thin lines at $z=1.5 \mathrm{~cm}$ and $T_{w}=0$ are also plotted as visual guides.

the other hand, the total thrust increases as the $Q_{\text {down }}$ ratio increases for a $B_{e x}$ of $200 \mathrm{G}$. In the recent experiment, the measured thrust increased with the increasing downstream gas injection ratio at a relatively high solenoid current. ${ }^{19}$ This result qualitatively agrees with the calculated total thrust for a $B_{e x}$ of $200 \mathrm{G}$. Therefore, the effect of downstream gas injection on thrust performance is effective only when a stronger magnetic field is applied to the thruster.

However, it should be noted that the simulations for charged particles and neutrals have been conducted separately. Hence, we do not consider consumption of neutrals when a high-density plasma is generated, or we do not incorporate the effect of neutral depletion in our simulations. Moreover, the thrust in the experiment included the thrust due to a magnetic nozzle in addition to $T_{w}$ and $T_{s}$. In order to discuss the relationship between the magnetic field and neutral distribution quantitatively, the neutral depletion effect and the calculation in the magnetic nozzle region have to be incorporated for comparison with the experiments in more detail, which remains a future issue in the development of the numerical code.
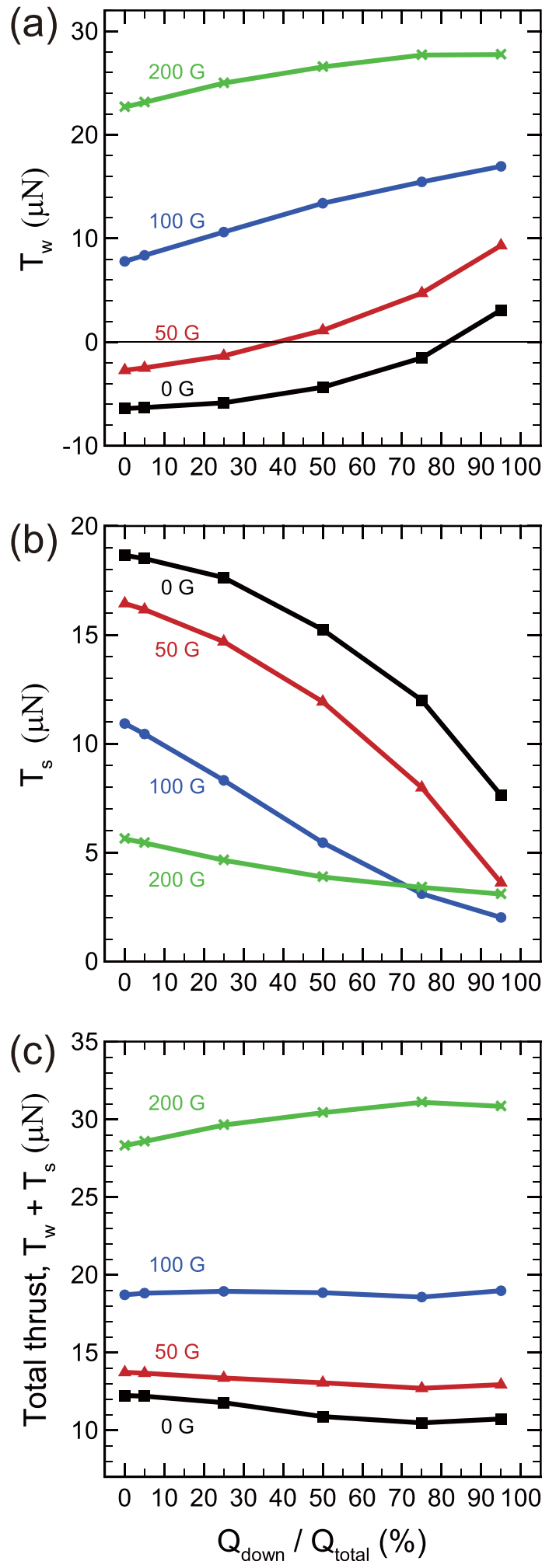

FIG. 7. Calculated thrust force on (a) the lateral wall $T_{w}$, (b) the back plate $T_{s}$, and (c) the total thrust (sum of $T_{w}$ and $T_{s}$ ) as a function of the downstream gas injection ratio $\left(Q_{\text {down }} / Q_{\text {total }}\right)$ for $B_{e x}$ of 0 (squares), 50 (triangles), 100 (circles), and $200 \mathrm{G}$ (crosses).

\section{CONCLUSIONS}

In this paper, we have conducted two-dimensional axisymmetric particle-in-cell simulations with a Monte Carlo collision algorithm (PIC-MCC) and the direct simulation Monte Carlo (DSMC) method in order to investigate the 
effects of neutral distribution and external magnetic field on the propulsion performance of electrodeless plasma thrusters. The numerical results show that the increase in downstream gas injection and application of an external magnetic field lead to shifts of the distributions of the plasma density and potential toward the downstream region. As a result, the thrust components and the total thrust are also affected by the downstream gas injection ratios and the external magnetic field, and an improvement of the thruster performance is shown when the downstream gas injection increases under the strong external magnetic field. This tendency is in qualitative agreement with the recent experimental results. Since the thrust of the electrodeless thruster is also affected by the Lorenz force onto the magnetic nozzle, an open boundary of the thruster exit is required for future PIC-MCC simulations.

\section{ACKNOWLEDGMENTS}

This work was partially supported by JSPS KAKENHI Grant No. JP16H04084 and JAXA. Part of the computer simulation was performed on the KDK computer system at Research Institute for Sustainable Humanosphere, Kyoto University.

${ }^{1}$ F. F. Chen, Plasma Sources Sci. Technol. 24, 014001 (2015).

${ }^{2}$ C. Schröder, O. Grulke, T. Klinger, and V. Naulin, Phys. Plasmas 11, 4249 (2004).

${ }^{3}$ R. W. Boswell, Phys. Lett. A 33, 457 (1970).

${ }^{4}$ P. Chabert, N. Proust, J. Perrin, and R. W. Boswell, Appl. Phys. Lett. 76, 2310 (2000)

${ }^{5}$ X. Wang, H. Masumoto, Y. Someno, and T. Hirai, Thin Solid Films 338, 105 (1999).

${ }^{6}$ C. Sarra-Bournet, C. Charles, and R. Boswell, Surf. Coat. Technol. 205, 3939 (2011).

${ }^{7}$ K. Takahashi, A. Chiba, A. Komuro, and A. Ando, Phys. Rev. Lett. 114, 195001 (2015).
${ }^{8}$ L. T. Williams and M. L. R. Walker, J. Propul. Power 29, 520 (2013).

${ }^{9}$ K. Takahashi, C. Charles, and R. W. Boswell, Phys. Rev. Lett. 110, 195003 (2013).

${ }^{10}$ A. Shabshelowitz and A. D. Gallimore, J. Propul. Power 29, 919 (2013).

${ }^{11}$ A. Fruchtman, Phys. Rev. Lett. 96, 065002 (2006).

${ }^{12}$ S. Pottinger, V. Lappas, C. Charles, and R. Boswell, J. Phys. D 44, 235201 (2011).

${ }^{13}$ Y. Takao and K. Takahashi, Phys. Plasmas 22, 113509 (2015).

${ }^{14}$ K. Takahashi, Y. Takao, and A. Ando, Appl. Phys. Lett. 108, 074103 (2016).

${ }^{15}$ R. M. Magee, M. E. Galante, J. Carr, Jr., G. Lusk, D. W. McCarren, and E. E. Scime, Phys. Plasmas 20, 123511 (2013).

${ }^{16}$ A. Aanesland, L. Liard, G. Leray, J. Jolly, and P. Chabert, Appl. Phys. Lett. 91, 121502 (2007).

${ }^{17}$ E. Ahedo and J. Navarro-Cavallé, Phys. Plasmas 20, 043512 (2013).

${ }^{18}$ A. Fruchtman, G. Makrinich, P. Chabert, and J. M. Rax, Phys. Rev. Lett. 95, 115002 (2005).

${ }^{19}$ K. Takahashi, Y. Takao, and A. Ando, Appl. Phys. Lett. 109, 194101 (2016).

${ }^{20}$ K. Takahashi and A. Ando, Plasma Phys. Controlled Fusion 59, 054007 (2017).

${ }^{21}$ H. Takekida and K. Nanbu, IEEE Trans. Plasma Sci. 34, 973 (2006).

${ }^{22}$ A. Fruchtman, K. Takahashi, C. Charles, and R. W. Boswell, Phys. Plasmas 19, 033507 (2012).

${ }^{23}$ K. Takahashi, T. Lafleur, C. Charles, P. Alexander, and R. W. Boswell, Phys. Rev. Lett. 107, 235001 (2011).

${ }^{24}$ K. Takahashi, T. Lafleur, C. Charles, P. Alexander, and R. W. Boswell, Phys. Plasmas 19, 083509 (2012).

${ }^{25}$ A. Fruchtman, IEEE Trans. Plasma Sci. 36, 403 (2008).

${ }^{26}$ Y. Takao, H. Koizumi, K. Komurasaki, K. Eriguchi, and K. Ono, Plasma Sources Sci. Technol. 23, 064004 (2014).

${ }^{27}$ Y. Takao, N. Kusaba, K. Eriguchi, and K. Ono, J. Appl. Phys. 108, 093309 (2010).

${ }^{28}$ H. Takekida and K. Nanbu, Thin Solid Films 506-507, 729 (2006).

${ }^{29}$ B. W. Yu and S. L. Girshick, J. Appl. Phys. 69, 656 (1991).

${ }^{30}$ X. Chen and E. Pfender, Plasma Chem. Plasma Process. 11, 103 (1991).

${ }^{31}$ C. K. Birdsall and A. B. Langdon, Plasma Physics via Computer Simulation (IOP Publishing, Bristol, UK, 1991).

${ }^{32}$ V. Vahedi and M. Surendra, Comput. Phys. Commun. 87, 179 (1995).

${ }^{33}$ J. P. Verboncoeur, J. Comput. Phys. 174, 421 (2001).

${ }^{34}$ M. Surendra and D. B. Graves, IEEE Trans. Plasma Sci. 19, 144 (1991).

${ }^{35}$ Y. Takao, K. Eriguchi, and K. Ono, J. Appl. Phys. 112, 093306 (2012). 